\title{
Evaluation of thromboelastometry parameters as predictive markers for sinusoidal obstruction syndrome in patients undergoing allogeneic stem cell transplantation for acute leukaemia
}

\author{
Joanna Rupa-Matysek ${ }^{1}$, Lidia Gil ${ }^{1}$, Ewelina Wojtasińska ${ }^{1}$, Zuzanna Kanduła ${ }^{1}$, Adam \\ Nowicki ${ }^{1}$, Magdalena Matuszak ${ }^{1}$ and Mieczysław Komarnicki ${ }^{1}$ \\ ${ }^{1}$ Department of Hematology and Bone Marrow Transplantation, Poznan University of Medical Sciences, Poznań, Poland \\ Correspondence to: Joanna Rupa-Matysek, email: rupa.matysek@gmail.com \\ Keywords: sinusoidal obstruction syndrome, acute leukemia, allogeneic stem cell transplantation, biomarkers, thrombo- \\ elastometry \\ Received: January 06, 2017 Accepted: May 29, $2017 \quad$ Published: June 15, 2017 \\ Copyright: Rupa-Matysek et al. This is an open-access article distributed under the terms of the Creative Commons Attribution License 3.0 (CC \\ BY 3.0), which permits unrestricted use, distribution, and reproduction in any medium, provided the original author and source are credited.
}

\section{ABSTRACT}

Hepatic sinusoidal obstruction syndrome (previously named veno-occlusive disease, SOS/VOD) is a serious complication of allogeneic stem cell transplantation (HSCT). Early identification of patients at risk of SOS/VOD may possibly improve the outcome and reduce mortality. Rotation thromboelastometry (ROTEM) is global assay allowing for the precise assessment of both bleeding and thrombotic conditions, however, its usefulness in patients undergoing HSCT for acute leukaemia has not been studied.

We evaluated the thromboelastometry parameters in patients undergoing allogeneic HSCT for acute leukaemia to identify candidate biomarkers of SOS/VOD occurrence.

ROTEM assays (INTEM, EXTEM, FIBTEM, APTEM) were performed on day $\mathbf{- 1 0}$, on the day of stem cell infusion (day 0 ) and on days +12 and +28 post-HSCT. The diagnosis of SOS/VOD was based on the Baltimore criteria. Seven patients $(26 \%)$ developed SOS/VOD. On day +12 , the patients with SOS/VOD had statistically significant longer INTEM-CT (clotting time, $199 \pm 33.41 \mathrm{vs} 166 \pm 23.65 s, p=0.0033$ ), EXTEM-CT (69.5 $\pm 6.39 v s .52 \pm 3.42 \mathrm{~s}, p=0.0139)$ and FIBTEM-CT (69.5 $\pm 22.75 \mathrm{vs}$. $50.8 \pm 14.31 s, p=0.0124)$ compared to SOS/VOD (-). ROC curve on day +12 indicated a cut-off value of 179s in INTEM-CT (AUC $=0.91), 69 \mathrm{~s}$ in EXTEM-CT $($ AUC $=0.90)$ and $102 \mathrm{~s}$ in FIBTEM-CT (AUC $=\mathbf{0 . 8 2}$ ) for the prediction of SOS/VOD.

This is the first study evaluating the usefulness of ROTEM assays in the early detection of haemostasis abnormalities predictive of SOS/VOD development in patients undergoing HSCT for acute leukemia. Patients with SOS/VOD had a significant delay in the initiation of thrombin formation in the analysed ROTEM assays. The utility of ROTEM assays in the optimal management of patients undergoing HSCT should be clarified in further prospective studies.

\section{INTRODUCTION}

Sinusoidal obstruction syndrome, previously called veno-occlusive disease of the liver (SOS/VOD), is a serious complication after haematopoietic stem cell transplantation (HSCT). SOS/VOD is more common after allogeneic HSCT (allo-HSCT) conditioned with a myeloablative regimen (MA), where it has an incidence of around $10-15 \%$ (ranging up to $40 \%$ in some studies), against $<5 \%$ after a reduced intensity conditioning regimen (RIC) and autologous HSCT $[1,2]$. The diagnosis of SOS/ VOD is based on clinical criteria including jaundice, painful hepatomegaly or ascites, and/or unexplained weight gain in a patient who fulfils either the modified Seattle 
criteria or the Baltimore criteria $[3,4]$. Despite the fact that the incidence rate of SOS/VOD is relatively low and most cases of mild SOS/VOD are self-limiting, a severe syndrome with multi-organ failure is still associated with a high mortality rate, reaching $80 \%[1,2,5]$. Therefore, early diagnosis and early treatment is essential to prevent disease progression to severe SOS/VOD and may possibly reduce mortality. The risk factors associated with increased risk of the development of SOS/VOD include patient-related factors such as age, underlying disease, liver damage, iron overload and previous/concomitant hepatotoxic drugs; and transplant-related factors; number of HSCT, type of transplant and donor, and the type of conditioning regimen the patient receives [5-7]. Preliminary data suggest that for early and accurate diagnosis of SOS, several potential biomarkers, or a panel of biological markers including those of endothelial injury or haemostatic parameters involved in SOS/VOD pathogenesis (especially plasminogen activator inhibitor-I, PAI-1), may be of some interest $[5,8]$.

The pathogenesis of SOS/VOD is complex and includes damage to endothelial cells and hepatocytes in zone 3 of the liver acinus. These are caused by toxic metabolites generated by the conditioning regimen, along with the cytokines released by the damaged tissues. All these processes lead to post-sinusoidal hypertension and the hepatorenal syndrome [5,9]. Changes in activity of coagulation factors due to endothelial cell injury and activation of fibrinolytic pathways not only contribute to SOS/VOD pathogenesis but may also be used to predict the occurrence of SOS/VOD [10, 11]. A hypercoagulable state during HSCT, profound thrombocytopenia and intravenous catheter all predispose to haemostasis abnormalities [12].

Thromboelastometry provides viscoelastic testing of haemostasis in whole blood and allows for the simultaneous evaluation of the different components involved in clot formation; plasma factors with contact activation and tissue factor activation, the contribution of platelets, fibrinogen and fibrinolysis [13].

The aim of the present study was to assess the coagulation abnormalities in patients treated for acute leukaemia with allogeneic HSCT by rotation thromboelastometry and to identify coagulopathy with respect to the development of SOS/VOD. The INTEM, EXTEM, FIBTEM and APTEM assays were all investigated.

\section{RESULTS}

\section{Patient characteristics}

This prospective observational study enrolled 27 adult patients, 14 males and 13 females. Median patient age was 38 years (range 18-63). Underlying diseases included acute myeloid leukaemia (AML, $n=20$ ) or acute lymphoblastic leukaemia (ALL, $n=7$ ). The patients underwent allogeneic stem cell transplantation from HLA-matched unrelated donors $(n=22)$ or sibling donors $(n=5)$ after myeloablative $(n=16)$ or reduced-intensity conditioning regimen $(n=11)$. Allogeneic peripheral blood stem cells $(n=16,59 \%)$, bone marrow $(n=10$, $37 \%)$, or cord blood $(n=1)$ were used as stem cell sources. The patients demographic and transplant characteristics are presented in Table 1.

All the patients engrafted in a median time of 21 days (range 15-29 days). Acute graft versus host disease (GvHD) was diagnosed in $22 \%$ of patients, while chronic GvHD developed in 11\%. In all the patients who developed acute graft versus host disease (aGvHD), the symptoms occurred 30 days post-HSCT.

\section{SOS/VOD}

All analysed patients had two or more risk factors for developing SOS/VOD according to published data and were considered as high risk. Seven patients developed SOS/VOD (26\%). Mild SOS/VOD occurred in $5(71 \%)$ patients and moderate in $2(29 \%)$ patients. None of them had a severe SOS/VOD or developed multiple organ failure. None of the patients who developed SOS/VOD died. The median time to SOS/VOD diagnosis after HSCT was 13 days (range 12-16). Among the aGVHD group, there was only one patient who developed mild SOS/ VOD with the disappearance of all SOS/VOD symptoms before the onset of aGVHD. Weight gain, jaundice and hepatomegaly were the most common manifestations. Therefore, the treatment was based on fluid restriction and diuretics and led to resolution of symptoms in all patients.

The patients with or without, symptoms of SOS/ VOD did not differ with regard to age, gender, type of acute leukaemia (AML versus ALL), type of donor (unrelated versus sibling), stem cell source (peripheral blood versus bone marrow stem cell) and intensity of conditioning. In those who received myeloablative regimen, no statistically significant differences were found between the patients conditioned with busulfan or TBI, Table 2.

The median ferritin level was significantly higher in the patients who developed SOS (3218, range 1352$4970 \mathrm{ng} / \mathrm{ml}$ ) in comparison to the patients who did not (598, range $152-3625 \mathrm{ng} / \mathrm{ml} ; p=0.0239$ ). Consistent with recent literature, our data show that a pre-transplant ferritin level above $1000 \mathrm{ng} / \mathrm{dl}$ was associated with SOS/ VOD development ( $p=0.0247)$, Table 2 .

\section{Coagulation parameters in the SOS/VOD patients}

No statistically significant differences in the APTT and the PT on day +12 , between the group with SOS/ VOD symptoms $(\mathrm{SOS} / \mathrm{VOD}+)$ and the group without SOS/VOD were found. Although the SOS/VOD (+) group 
Table 1: Clinical characteristics of patients and grafts

Parameter

Number of patients

Gender, female/male

Age; median, range years

\section{Diagnosis/Disease}

AML

ALL

Donor type

- sibling

- unrelated

Stem cell source

- peripheral blood

- bone marrow

- cord blood

Conditioning regimens

- myeloablative (MA)

$\mathrm{BuCy}$

TBICy

- reduced-intensity (RIC)

Fludarabine-based regimen

FluMel

FluBu2

\section{GvHD prophylaxis}

- MTX+CsA

- $\mathrm{MTX}+\mathrm{Cs} \mathrm{A}+\mathrm{ATG}$

Other risk factors

Second HSCT

Advanced disease (beyond second CR or relapse)

Iron overload (ferritin $>1000 \mathrm{ng} / \mathrm{ml}$ )

Previous liver disease

\section{Complications}

- Acute GvHD

- Chronic GvHD

- SOS/VOD

- Gram negative bloodstream infections

- Central venous catheter-related thrombosis

- Serious bleeding event

- Organ toxicity grade 3 or 4

\section{$n$}

27

$13 / 14$

$38(18-63)$

$$
20
$$

7

5

22

16

10

1

16

9

7

11

11

6

5

5

22

7

5

7

2

6

3

7

4

1

0

0

Abbreviations: AML - acute myeloid leukaemia, ALL - acute lymphoblastic leukaemia, ATG - anti-thymocyte globulin, $\mathrm{BuCy}$ - busulfan plus cyclophosphamide, CsA - ciclosporin, FluMel - fludarabine plus melphalan, GvHD - graft versus host disease, MAC - myeloablative conditioning regimen, MTX - methotrexate, RIC - reduced-intensity conditioning regimen, SOS/VOD - sinusoidal obstraction syndrome, TBI Cy - total body irradiation plus cyclophosphamide.

had a higher median plasma fibrinogen concentration, no marked differences in the median fibrinogen level were observed at the time of SOS/VOD occurrence between the $\mathrm{SOS} / \mathrm{VOD}(+)$ and SOS/VOD (-) groups. As in all HSCT 
Table 2: Clinical characteristics of the SOS/VOD positive and negative patients

\begin{tabular}{lccc}
\hline \multicolumn{1}{c}{ Risk factors } & SOS/VOD (+) & SOS/VOD (-) & \multirow{2}{*}{$\boldsymbol{p}$ value } \\
\cline { 2 - 3 } \multicolumn{1}{c}{ Transplant-related factors } & $\boldsymbol{n = 7}$ & $\boldsymbol{n}=\mathbf{2 0}$ & \\
Myeloablative conditioning (TBICy+BuCy) & $4(57 \%)$ & $12(60 \%)$ & 0.8946 \\
BuCy & $1(14 \%)$ & $8(40 \%)$ & 0.2142 \\
TBICy & $3(43 \%)$ & $4(20 \%)$ & 0.2349 \\
Unrelated donor & $7(100 \%)$ & $15(75 \%)$ & 0.1427 \\
Second HSCT & $1(14 \%)$ & $6(30 \%)$ & 0.4142 \\
$\quad$ Patient- and disease-related factors & & & \\
Age $>$ median 38 years & $3(43 \%)$ & $11(55 \%)$ & 0.5800 \\
Female gender & $2(28 \%)$ & $11(55 \%)$ & 0.2284 \\
AML & $4(57 \%)$ & $16(80 \%)$ & 0.2349 \\
Advanced disease (beyond second CR or relapse) & $2(29 \%)$ & $3(15 \%)$ & 0.4263 \\
Iron overload (ferritin $>1000 \mathrm{ng} / \mathrm{ml})$ & $6(75 \%)$ & $1(5 \%)$ & 0.0247 \\
Previous liver disease & $1(14 \%)$ & $1(5 \%)$ & 0.4195 \\
\hline Abbrian
\end{tabular}

Abbreviations: BuCy - busulfan plus cyclophosphamide, TBI Cy - total body irradiation plus cyclophosphamide. $p$ value between SOS $(+) /$ VOD and SOS/VOD $(-)$ group $>0.05-$ not significant. $p$ value $<0.05-$ statistically significant.

Table 3: Platelet and plasma coagulation laboratory results from HSCT recipients with or without SOS/VOD during the study on day +12 after HSCT

\begin{tabular}{|c|c|c|c|}
\hline \multirow{2}{*}{ Parameter } & SOS/VOD (+) & SOS/VOD (-) & \multirow{2}{*}{$p$ value } \\
\hline & \multicolumn{2}{|c|}{$\mathrm{ME} \pm \mathrm{SE}(\mathrm{Q} 1 ; \mathrm{Q3})$} & \\
\hline APTT $[\mathrm{s}]$ & $34.4 \pm 3.62(33.3-43.8)$ & $34.3 \pm 8.22(30.85-45.15)$ & 0.973 \\
\hline $\mathrm{PT}[\mathrm{s}]$ & $16.2 \pm 2.34(13.1-15.2)$ & $14.25 \pm 4.69(12.7-15.15)$ & 0.734 \\
\hline Platelet count $\left[\times 10^{9} / 1\right]$ & $22.0 \pm 2.70(20.0-29.0)$ & $22.66 \pm 4.70(16.8-34.55)$ & 0.786 \\
\hline Fibrinogen $[\mathrm{g} / \mathrm{l}]$ & $4.55 \pm 0.36(4.19-4.92)$ & $3.14 \pm 0.25(2.37-4.16)$ & 0.106 \\
\hline
\end{tabular}

Abbreviations: APTT - activated partial tromboplastin time [s]; PT - prothrombin time [s].

Data are expressed as median \pm standard error (interquartile range) - $\mathrm{Me} \pm \mathrm{SE}(\mathrm{Q} 1 ; \mathrm{Q} 3)$.

$p$ value $>0.05-$ not significant.

recipients, the platelet count was below the reference range on day +12 and no significant reductions in the platelet count were seen in the patients with symptoms of SOS/VOD, Table 3.

\section{ROTEM parameters in the SOS/VOD group}

Parameters concerning initiation and speed at which a solid clot forms (CT, CFT, $\alpha$-angle)

Compared with patients without SOS/VOD, the SOS/VOD $(+)$ group had significantly longer times between the activator being added and initial clot formation (coagulation time, CT) on day +12 in the
INTEM $(\mathrm{Me} \pm \mathrm{SE}, 199 \pm 33.41$ versus $166 \pm 23.65 \mathrm{~s}$, $p=0.0033)$, EXTEM $(69.5 \pm 6.39$ versus $52 \pm 3.42 \mathrm{~s}$, $p=0.0139)$ and FIBTEM $(69.5 \pm 22.7$ versus $50.8 \pm 14.31 \mathrm{~s}$, $p=0.0124)$ assays, Figure 1 .

In the SOS/VOD (+) group, no significant differences were found in the clot formation time (CFT), defined as the interval between the onset of coagulation and the clot reaching an amplitude of $20 \mathrm{~mm}$, and the values of $\alpha$-angle, referring to the steepness of the curves, in comparison to the patients without SOS/VOD. The thromboelastometry values are shown in Table 4.

To sum up, these results suggest that the SOS/ VOD $(+)$ patients had a tendency to delayed activation of 
Table 4: Comparison of standard ROTEM profiles between the SOS/VOD (+) group and the SOS/ VOD (-) group

\begin{tabular}{|c|c|c|c|c|c|c|c|}
\hline $\begin{array}{l}\text { ROTEM } \\
\text { data }\end{array}$ & \multicolumn{3}{|c|}{$\begin{array}{l}\mathrm{Me} \pm \mathrm{SE}(\mathrm{Q} 1 ; \mathrm{Q3}) \\
\text { day +12 after transplantation }\end{array}$} & \multicolumn{4}{|c|}{$\begin{array}{l}\text { Me } \pm \mathrm{SE}(\mathrm{Q} 1 ; \mathrm{Q3}) \\
\text { cansplantation }\end{array}$} \\
\hline \multirow{9}{*}{$\begin{array}{l}\sum_{\text {c }} \\
\text { 点 }\end{array}$} & CT & $\begin{array}{c}199 \pm 33.41 \\
(191 ; 237)\end{array}$ & $\begin{array}{l}166 \pm 23.65 \\
(166 ; 175)\end{array}$ & 0.0033 & $\begin{array}{c}193.5 \pm 17.82 \\
(178 ; 224.5)\end{array}$ & $\begin{array}{l}173 \pm 6.94 \\
(160 ; 185)\end{array}$ & 0.2373 \\
\hline & CFT & $\begin{array}{l}110 \pm 30.91 \\
(71.5 ; 165)\end{array}$ & $\begin{array}{c}131 \pm 22.36 \\
(73 ; 203)\end{array}$ & 0.5304 & $\begin{array}{c}162.5 \pm 53.56 \\
(132.5 ; 275)\end{array}$ & $\begin{array}{c}82 \pm 25.03 \\
(72 ; 144)\end{array}$ & 0.1199 \\
\hline & $\begin{array}{c}\text { Alfa } \\
\text { angle }\end{array}$ & $\begin{array}{l}78.5 \pm 0.65 \\
(77.5 ; 79.5)\end{array}$ & $\begin{array}{l}78 \pm 0.73 \\
(75 ; 80)\end{array}$ & 0.6646 & $\begin{array}{l}73 \pm 6.07 \\
(63 ; 78.5)\end{array}$ & $\begin{array}{l}76 \pm 0.76 \\
(74 ; 78)\end{array}$ & 0.3168 \\
\hline & A10 & $\begin{array}{c}35.5 \pm 2.95 \\
(32 ; 40.5)\end{array}$ & $\begin{array}{l}35 \pm 1.91 \\
(29 ; 40)\end{array}$ & 0.6646 & $\begin{array}{c}29.5 \pm 2.56 \\
(26.5 ; 34)\end{array}$ & $\begin{array}{l}51 \pm 2.96 \\
(36 ; 54)\end{array}$ & 0.0404 \\
\hline & A15 & $\begin{array}{l}39 \pm 2.32 \\
(36.5 ; 43)\end{array}$ & $\begin{array}{c}38 \pm 1.92 \\
(32 ; 42)\end{array}$ & 0.4107 & $\begin{array}{c}33.5 \pm 2.9 \\
(30 ; 39)\end{array}$ & $\begin{array}{l}55 \pm 3.08 \\
(41 ; 59)\end{array}$ & 0.0404 \\
\hline & A20 & $\begin{array}{c}42 \pm 2.48 \\
(38.5 ; 45.5)\end{array}$ & $\begin{array}{c}40 \pm 1.83 \\
(35 ; 45)\end{array}$ & 0.4107 & $\begin{array}{c}35.5 \pm 2.75 \\
(32.5 ; 41)\end{array}$ & $\begin{array}{c}57 \pm 2.83 \\
(43 ; 59)\end{array}$ & 0.0518 \\
\hline & A25 & $\begin{array}{c}43 \pm 2.48 \\
(39.5 ; 46.5)\end{array}$ & $\begin{array}{c}42 \pm 1.81 \\
(37 ; 46)\end{array}$ & 0.5304 & $\begin{array}{l}37 \pm 3.01 \\
(33.5 ; 43)\end{array}$ & $\begin{array}{c}58 \pm 2.72 \\
(45 ; 60)\end{array}$ & 0.0518 \\
\hline & MCF & $\begin{array}{c}43.5 \pm 2.46 \\
(40.5 ; 47)\end{array}$ & $\begin{array}{c}45 \pm 4.24 \\
(38 ; 52)\end{array}$ & 0.8096 & $\begin{array}{l}41 \pm 2.39 \\
(39 ; 45.5)\end{array}$ & $\begin{array}{c}58 \pm 2.63 \\
(46 ; 60)\end{array}$ & 0.0805 \\
\hline & ML & $\begin{array}{c}12 \pm 0.57 \\
(11 ; 12)\end{array}$ & $\begin{array}{c}12 \pm 0.22 \\
(12 ; 12)\end{array}$ & 0.5067 & $\begin{array}{c}10 \pm 0.86 \\
(8 ; 11)\end{array}$ & $\begin{array}{l}9.5 \pm 0.61 \\
(7.5 ; 11.5)\end{array}$ & 0.9559 \\
\hline \multirow{9}{*}{$\sum_{\substack{x \\
y}}$} & CT & $\begin{array}{c}69.5 \pm 6.39 \\
(63 ; 79)\end{array}$ & $\begin{array}{c}52 \pm 3.42 \\
(47 ; 60)\end{array}$ & 0.0139 & $\begin{array}{c}56 \pm 6.56 \\
(52 ; 68)\end{array}$ & $\begin{array}{c}49 \pm 2.49 \\
(46 ; 50)\end{array}$ & 0.0805 \\
\hline & CFT & $\begin{array}{c}105 \pm 26.76 \\
(70.5 ; 143.5)\end{array}$ & $\begin{array}{c}141 \pm 24.06 \\
(79 ; 213)\end{array}$ & 0.4107 & $\begin{array}{c}132 \pm 64.3 \\
(117.5 \\
262.5)\end{array}$ & $\begin{array}{l}94 \pm 23.2 \\
(64 ; 162)\end{array}$ & 0.2373 \\
\hline & $\begin{array}{l}\text { Alfa } \\
\text { angle }\end{array}$ & $\begin{array}{l}80 \pm 0.75 \\
(78.5 ; 81)\end{array}$ & $\begin{array}{c}79 \pm 0.79 \\
(76 ; 81)\end{array}$ & 0.4690 & $\begin{array}{c}79 \pm 7.18 \\
(65 ; 80)\end{array}$ & $\begin{array}{c}77 \pm 1.18 \\
(73 ; 81)\end{array}$ & 0.8291 \\
\hline & A10 & $\begin{array}{c}36.5 \pm 2.06 \\
(34 ; 39.5)\end{array}$ & $\begin{array}{c}35 \pm 1.97 \\
(32 ; 40)\end{array}$ & 0.8096 & $\begin{array}{c}33 \pm 3.49 \\
(27.5 ; 38.5)\end{array}$ & $\begin{array}{c}52 \pm 2.95 \\
(38 ; 56)\end{array}$ & 0.0648 \\
\hline & A15 & $\begin{array}{l}39.5 \pm 1.87 \\
(37.5 ; 42.5)\end{array}$ & $\begin{array}{c}38 \pm 1.91 \\
(35 ; 44)\end{array}$ & 0.5965 & $\begin{array}{c}37 \pm 3.65 \\
(31 ; 43)\end{array}$ & $\begin{array}{c}57 \pm 2.94 \\
(42 ; 59)\end{array}$ & 0.0648 \\
\hline & A20 & $\begin{array}{c}41 \pm 2.12 \\
(39.5 ; 44.5)\end{array}$ & $\begin{array}{c}41 \pm 1.83 \\
(37 ; 46)\end{array}$ & 0.8096 & $\begin{array}{l}39.5 \pm 3.57 \\
(33.5 ; 45.5)\end{array}$ & $\begin{array}{c}59 \pm 2.85 \\
(45 ; 61)\end{array}$ & 0.0648 \\
\hline & A25 & $\begin{array}{c}42 \pm 1.73 \\
(41 ; 45)\end{array}$ & $\begin{array}{c}42 \pm 1.78 \\
(39 ; 48)\end{array}$ & 0.8854 & $\begin{array}{l}41 \pm 3.47 \\
(35.5 ; 47)\end{array}$ & $\begin{array}{c}60 \pm 2.77 \\
(46 ; 61)\end{array}$ & 0.0805 \\
\hline & MCF & $\begin{array}{l}44 \pm 1.84 \\
(42 ; 46.5)\end{array}$ & $\begin{array}{c}43 \pm 2.98 \\
(40 ; 50)\end{array}$ & 0.9613 & $\begin{array}{c}43.5 \pm 2.92 \\
(39 ; 49)\end{array}$ & $\begin{array}{c}61 \pm 2.62 \\
(47 ; 62)\end{array}$ & 0.1199 \\
\hline & ML & $\begin{array}{c}10 \pm 0.26 \\
(10 ; 10)\end{array}$ & $\begin{array}{c}10 \pm 0.145 \\
(10 ; 10)\end{array}$ & 0.6185 & $\begin{array}{c}12 \pm 0.84 \\
(8 ; 12)\end{array}$ & $\begin{array}{c}12 \pm 0.39 \\
(10 ; 12)\end{array}$ & 0.9558 \\
\hline
\end{tabular}




\begin{tabular}{|c|c|c|c|c|c|c|c|}
\hline \multirow{4}{*}{ 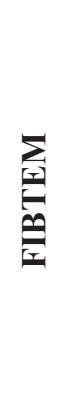 } & CT & $\begin{array}{c}69.5 \pm 22.75 \\
(55.5 ; 83.5)\end{array}$ & $\begin{array}{c}50.8 \pm 14.31 \\
(40 ; 61)\end{array}$ & 0.0124 & $\begin{array}{c}50 \pm 7.71 \\
(40.5 ; 63.5)\end{array}$ & $\begin{array}{c}43.5 \pm 3.13 \\
\quad(40 ; 50)\end{array}$ & 0.6167 \\
\hline & A10 & $\begin{array}{c}26.5 \pm 5.01 \\
(22 ; 34.5)\end{array}$ & $\begin{array}{l}25 \pm 1.4 \\
(19 ; 31)\end{array}$ & 0.7363 & $\begin{array}{l}22 \pm 3.12 \\
(17 ; 25.5)\end{array}$ & $\begin{array}{l}21 \pm 1.43 \\
(17.5 ; 27)\end{array}$ & 0.9635 \\
\hline & A20 & $\begin{array}{l}28.5 \pm 5.18 \\
(23.5 ; 36.5)\end{array}$ & $\begin{array}{c}27 \pm 1.86 \\
(20 ; 33)\end{array}$ & 0.6646 & $\begin{array}{c}23 \pm 3.5 \\
(18 ; 27.5)\end{array}$ & $\begin{array}{l}23 \pm 1.53 \\
(18.5 ; 29)\end{array}$ & 0.9634 \\
\hline & MCF & $\begin{array}{l}30 \pm 5.57 \\
(24 ; 38.5)\end{array}$ & $\begin{array}{c}30 \pm 3.99 \\
(21 ; 37)\end{array}$ & 0.9613 & $\begin{array}{c}23.5 \pm 3.33 \\
(18.5 ; 28)\end{array}$ & $\begin{array}{l}23.5 \pm 1.57 \\
(18.5 ; 29.5)\end{array}$ & 0.9635 \\
\hline \multirow{5}{*}{$\frac{\sum_{\text {S }}}{2}$} & CT & $\begin{array}{c}60 \pm 21.67 \\
(25 ; 100)\end{array}$ & $\begin{array}{r}53 \pm 5.12 \\
(47 ; 58)\end{array}$ & 0.8000 & $\begin{array}{c}54.5 \pm 9.78 \\
(47.5 ; 75)\end{array}$ & $\begin{array}{c}56.5 \pm 3.03 \\
(44 ; 59.5)\end{array}$ & 0.3352 \\
\hline & A10 & $\begin{array}{c}105 \pm 36.04 \\
(67 ; 189)\end{array}$ & $\begin{array}{c}129 \pm 21.29 \\
(81 ; 181)\end{array}$ & 0.8000 & $\begin{array}{l}151 \pm 58.08 \\
(130 ; 263.5)\end{array}$ & $\begin{array}{c}119 \pm 31.47 \\
(73 ; 193)\end{array}$ & 0.2902 \\
\hline & A20 & $\begin{array}{c}78 \pm 0.88 \\
(77 ; 80)\end{array}$ & $\begin{array}{c}77 \pm 2.84 \\
(75 ; 78)\end{array}$ & 0.3642 & $\begin{array}{l}80 \pm 5.5 \\
(69 ; 80)\end{array}$ & $\begin{array}{c}77.5 \pm 2.09 \\
(70.5 ; 80)\end{array}$ & 0.7505 \\
\hline & MCF & $\begin{array}{r}44 \pm 3.61 \\
(41 ; 53)\end{array}$ & $\begin{array}{c}45 \pm 3.52 \\
(40 ; 53)\end{array}$ & 0.9999 & $\begin{array}{c}43.5 \pm 2.69 \\
(40 ; 48.5)\end{array}$ & $\begin{array}{l}54 \pm 2.75 \\
(44.5 ; 62)\end{array}$ & 0.1482 \\
\hline & ML & $\begin{array}{c}10 \pm 0.55 \\
(10 ; 10)\end{array}$ & $\begin{array}{c}10 \pm 0.11 \\
(10 ; 10)\end{array}$ & 0.7821 & $\begin{array}{c}10 \pm 0.42 \\
(10 ; 11)\end{array}$ & $\begin{array}{r}10 \pm 0.15 \\
(10 ; 10)\end{array}$ & 0.0722 \\
\hline
\end{tabular}

Abbreviations: CT - clotting time [s], CFT - clot formation time [s], alfa-angle - steepness of curve [degree (o)], MCF maximum clot firmness [mm], ML - maximum lysis [\%], A10-A25 - firmness at time 10-25 minutes [mm].

Data are expressed as median \pm standard error (interquartile range) - $\mathrm{Me} \pm \mathrm{SE}(\mathrm{Q} 1 ; \mathrm{Q} 3) p$ value $\geq 0.05$ - not significant. $p$ value $<0.05-$ statistically significant.
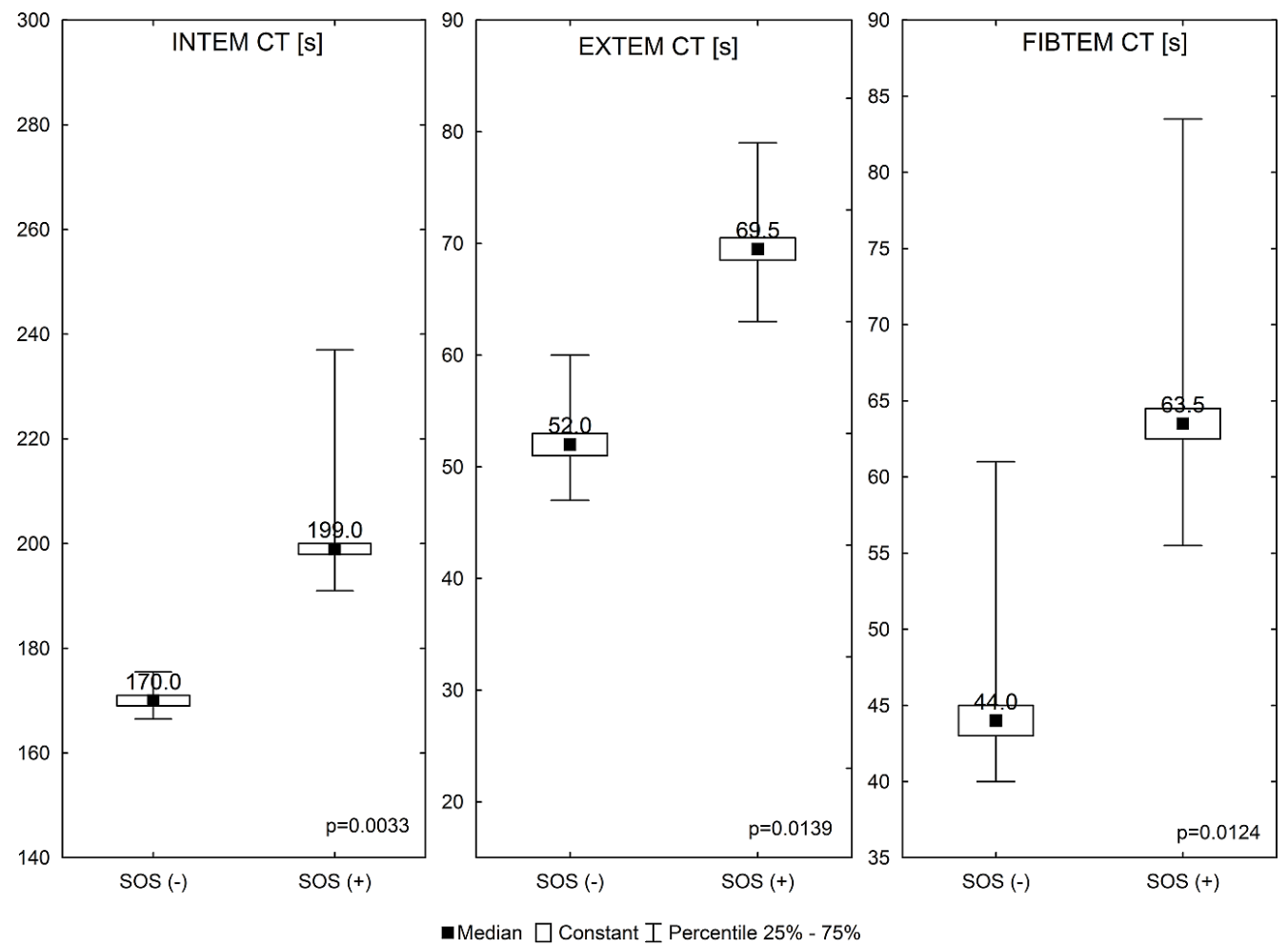

Figure 1: Comparison of selected ROTEM parameters between the SOS/VOD (+) group and SOS/VOD (-) group. Abbreviations: CT - clotting time [s]. 
haemostasis with prolonged initial clot formation in the INTEM, EXTEM and FIBTEM assays.

\section{Parameters concerning clot firmness (MCF, A10, A15, A20, A25)}

Analyses of the parameters concerning clot firmness, including maximum clot firmness (MCF), amplitude at 10, 15, 20 and 25 minutes (A10, A15, A20 and A25), did not differ between the patients with or without SOS/ VOD on day +12 (Table 4). In summary, in our study the parameters concerning clot strength were unsuitable for the identification of SOS/VOD on day +12 in patients undergoing HSCT.

On day +28 , INTEM-A10 and INTEM-A15 were significantly different between SOS/VOD (+) and SOS/ VOD (-) indicating decreased clot firmness in the SOS/ VOD (+) group. Other parameters related to the initiation of clotting (CT), fibrin polymerisation (CFT), stabilisation of the clot by fibrin and thrombocytes (MCF) and stability of the clot (ML) in all analysed assays did not differ statistically between the patients with or without SOS/ VOD on day +28 .

\section{Parameters concerning clot lysis (ML, LI30, LI45 and LI60)}

No differences were found in the parameters concerning clot lysis, including the maximum lysis and the lysis indices at 30,45 and 60 minutes, between the SOS/VOD (+) and SOS/VOD (-) group. Therefore, the values of parameters concerning clot lysis did not assist in distinguishing the SOS/VOD (+) group from the patients without SOS/VOD symptoms.

\section{Parameters concerning velocity profile (MaxVel, t-MaxVel and AUC)}

The values of maximum velocity (MaxVel) did not differ in the SOS/VOD (+) group from those in the SOS/ VOD (-) group. Except for a longer time to maximum velocity (t-MaxVel) in EXTEM on day +12 in the SOS/ VOD $(+)$ patients, no significant differences were found in results of t-MaxVel. Moreover, no differences in the area under the 1st derivative curve (AUC) were found between the analysed groups.

To summarise, our findings suggest that the velocity parameters were not able to differentiate between patients with or without SOS/VOD symptoms. Detailed data concerning the thromboelastometry values in patients with or without SOS/VOD are shown in Table 5.

\section{Cut-off values of CT for the prediction of SOS/ VOD}

ROC analysis indicated a cut-off value of $179 \mathrm{~s}$ in INTEM-CT $(\mathrm{AUC}=0.91 ; 95 \%$ CI $0.72-1.0, \mathrm{SE}=0.088)$, $69 \mathrm{~s}$ in EXTEM-CT (AUC $=0.90 ; 95 \%$ CI $0.75-1.0$, $\mathrm{SE}=0.076)$ and $102 \mathrm{~s}$ in FIBTEM-CT $(\mathrm{AUC}=0.82 ; 95 \%$
CI $0.61-1.0, \mathrm{SE}=0.104)$ for the prediction of SOS/VOD in patients undergoing HSCT on day +12 . ROC curves of INTEM-CT, EXTEM-CT and FIBTEM-CT for SOS/VOD prediction are provided in Figure 2.

\section{Other complications in the early post-transplant period}

None of the patients developed a bleeding episode considered life threatening. In one patient, central catheterrelated thrombosis was documented on day +5 after transplantation.

Gram-negative blood-stream infections (GNBSI), including Klebsiella pneumonia, Enterobacter cloacae complex, Enterobacter aerogenes and Citobacter koseri were found in four patients. The small sample size did not allow for thromboelastometry subanalyses.

\section{DISCUSSION}

To our knowledge, this is the first analysis of haemostasis abnormalities in patients undergoing allogeneic HSCT for acute leukemia by rotation thromboelastometry together with an assessment of coagulation dynamic properties with respect to the occurrence of SOS/VOD.

The pathogenesis of SOS/VOD is complex, including endothelial injury and increased expression of adhesion molecules, pro-inflammatory cytokines and procoagulant factors [23-30]. A procoagulant state with a decreased level of antithrombin and protein $\mathrm{C}$, consumption of factor VII, and an increased level of plasminogen activator inhibitor-1 (PAI-1) has been demonstrated at the time of SOS/VOD diagnosis in many studies [10-12, 31-34]. The level of PAI-1 is also important as a prognostic marker [10, 23, 35-38]. Studies are on-going including a quantitative mass spectrometry-based proteomics approach to identify candidate biomarkers of SOS/VOD [8]. Most of the biomarkers which have been reported so far as candidate clinical markers for SOS/VOD are not readily available or have only been examined in a limited number of patients making the results inconclusive.

Rotational thromboelastometry (ROTEM) enables the measurement of global clot formation and dissolution in whole blood in real time [39]. In whole blood, the interactions of coagulation factors, platelets, and fibrinogen with coagulation inhibitors during clot formation and subsequent fibrinolysis are analysed by ROTEM. The primary clinical application of ROTEM assays is in the assessment of haemostasis abnormalities to treat bleeding patients [39] as recommended in transfusion algorithms. Other studies indicate that perioperative ROTEM assays may detect patients at risk for postoperative thromboembolic complications [40]. As ROTEM assays allow for the assessment of 
Table 5: Comparison of coagulation dynamic properties analysed by ROTEM between the SOS/ VOD (+) group and the SOS/VOD (-) group

\begin{tabular}{|c|c|c|c|c|c|c|c|}
\hline \multirow{2}{*}{\multicolumn{2}{|c|}{ ROTEM data }} & SOS/VOD $(+)$ & SOS/VOD (-) & \multirow{2}{*}{$p$ value } & SOS/VOD $(+)$ & SOS/VOD (-) & \multirow[b]{2}{*}{$p$ value } \\
\hline & & \multicolumn{2}{|c|}{$\begin{array}{l}\mathrm{Me} \pm \mathrm{SE}(\mathrm{Q} 1 ; \mathrm{Q3}) \\
\text { day }+12 \text { after transplantation }\end{array}$} & & \multicolumn{2}{|c|}{$\begin{array}{l}\text { Me } \pm \text { SE }(\mathrm{Q} 1 ; \mathrm{Q3}) \\
\text { day }+28 \text { after transplantation }\end{array}$} & \\
\hline \multirow{3}{*}{ 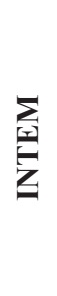 } & AUC & $\begin{array}{c}4341.05 \pm 138.34 \\
(4258 ; 4354)\end{array}$ & $\begin{array}{c}4341.05 \pm 138.91 \\
(3919 ; 4547)\end{array}$ & 0.6646 & $\begin{array}{c}4946 \pm 71.4 \\
(3812 ; 7636)\end{array}$ & $\begin{array}{c}5914.5 \pm 2794.41 \\
(4619 ; 6489)\end{array}$ & 0.0805 \\
\hline & t-MaxV & $\begin{array}{c}208.4 \pm 14.8 \\
(192 ; 246)\end{array}$ & $\begin{array}{c}205.7 \pm 7.88 \\
(182 ; 209)\end{array}$ & 0.8854 & $\begin{array}{l}198 \pm 11.4 \\
(198 ; 204)\end{array}$ & $\begin{array}{l}193 \pm 6.38 \\
(178 ; 202)\end{array}$ & 0.2158 \\
\hline & MaxV & $\begin{array}{c}21.47 \pm 0.96 \\
(19 ; 24)\end{array}$ & $\begin{array}{c}21.47 \pm 1.19 \\
(19 ; 22)\end{array}$ & 0.7363 & $\begin{array}{c}20.0 \pm 1.98 \\
(17 ; 20)\end{array}$ & $\begin{array}{l}19.5 \pm 1.13 \\
(16 ; 22)\end{array}$ & 0.8421 \\
\hline \multirow{3}{*}{$\sum_{\substack{x=1 \\
=}}$} & AUC & $\begin{array}{c}4428.47 \pm 94.11 \\
(4347 ; 4428)\end{array}$ & $\begin{array}{l}4428.47 \pm 129.21 \\
\quad(4190 ; 4554)\end{array}$ & 0.9613 & $\begin{array}{c}4926 \pm 225.32 \\
(3837 ; 5140)\end{array}$ & $\begin{array}{c}5140.85 \pm 5140.86 \\
(4644 ; 6043)\end{array}$ & 0.1200 \\
\hline & t-MaxV & $\begin{array}{c}68.0 \pm 5.01 \\
(66 ; 91)\end{array}$ & $\begin{array}{c}61.0 \pm 2.42 \\
(55 ; 66)\end{array}$ & 0.0139 & $\begin{array}{c}64.2 \pm 3.96 \\
(61 ; 67)\end{array}$ & $\begin{array}{l}64.2 \pm 64.24 \\
(55 ; 64)\end{array}$ & 0.4618 \\
\hline & $\operatorname{Max} V$ & $\begin{array}{c}23.16 \pm 1.08 \\
(22 ; 26)\end{array}$ & $\begin{array}{c}23.15 \pm 1.05 \\
(19 ; 25)\end{array}$ & 0.7363 & $\begin{array}{c}19.76 \pm 1.98 \\
(19 ; 22)\end{array}$ & $\begin{array}{c}19.76 \pm 19.76 \\
(16 ; 22)\end{array}$ & 0.8291 \\
\hline \multirow{3}{*}{ 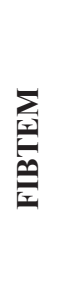 } & $\mathbf{A U C}$ & $\begin{array}{c}2952.84 \pm 302.39 \\
(2757 ; 3126)\end{array}$ & $\begin{array}{l}2952.84 \pm 149.21 \\
(2556 ; 3404)\end{array}$ & 0.8855 & $\begin{array}{l}2320.35 \pm 185.05 \\
\quad(2136 ; 2512)\end{array}$ & $\begin{array}{c}2320.35 \pm 123.38 \\
(2008.5 ; 2640)\end{array}$ & 0.9635 \\
\hline & t-MaxV & $\begin{array}{c}64.9 \pm 7.72 \\
(64 ; 86)\end{array}$ & $\begin{array}{c}64.9 \pm 3.52 \\
(47 ; 66)\end{array}$ & 0.0624 & $\begin{array}{c}56.3 \pm 4.25 \\
(55 ; 64)\end{array}$ & $\begin{array}{c}53.5 \pm 2.57 \\
(49 ; 56)\end{array}$ & 0.5536 \\
\hline & $\operatorname{MaxV}$ & $\begin{array}{l}28.16 \pm 2.79 \\
\quad(24 ; 28)\end{array}$ & $\begin{array}{l}28.15 \pm 1.15 \\
\quad(25 ; 31)\end{array}$ & 0.6646 & $\begin{array}{l}26.05 \pm 2.1 \\
(26 ; 27)\end{array}$ & $\begin{array}{c}26.03 \pm 2.04 \\
(22 ; 30)\end{array}$ & 0.8916 \\
\hline \multirow{3}{*}{$\sum_{\substack{x \\
2}}$} & AUC & $\begin{array}{c}6798.24 \pm 476.45 \\
(4434 ; 6798)\end{array}$ & $\begin{array}{c}5649 \pm 1721.97 \\
(4455 ; 6798)\end{array}$ & 0.6765 & $\begin{array}{c}4842.5 \pm 154.56 \\
(4033 ; 4842)\end{array}$ & $\begin{array}{c}4842.5 \pm 195.41 \\
(4561 ; 5535)\end{array}$ & 0.2902 \\
\hline & t-MaxV & $\begin{array}{c}69.5 \pm 6.46 \\
(69 ; 79)\end{array}$ & $\begin{array}{c}68.23 \pm 5.52 \\
(57 ; 69)\end{array}$ & 0.0676 & $\begin{array}{c}63.7 \pm 4.89 \\
(61 ; 67)\end{array}$ & $\begin{array}{c}62 \pm 3.06 \\
(53 ; 65)\end{array}$ & 0.5536 \\
\hline & $\operatorname{MaxV}$ & $\begin{array}{c}23.53 \pm 2.29 \\
(23 ; 24)\end{array}$ & $\begin{array}{c}23.52 \pm 1.12 \\
(20 ; 26)\end{array}$ & 0.4323 & $\begin{array}{c}19.25 \pm 2.26 \\
(19 ; 25)\end{array}$ & $\begin{array}{c}19.25 \pm 1.11 \\
(14 ; 23)\end{array}$ & 0.2902 \\
\hline
\end{tabular}

Abbreviations: MaxVel - maximum velocity [mm/min], MaxVel-t - time to maximum velocity [s], AUC - area under 1st derivative curve $[\mathrm{mm} \times 100]$.

Data are expressed as median \pm standard error (interquartile range) - $\mathrm{Me} \pm \mathrm{SE}(\mathrm{Q} 1 ; \mathrm{Q} 3) p$ value $\geq 0.05$ - not significant.

$p$ value $<0.05-$ statistically significant.

both bleeding and thrombotic conditions, we evaluated their application in patients undergoing HSCT for acute leukaemia. So far, no information is available on the utility of thromboelastometry in the identification of patients at risk of SOS/VOD development. In addition, no data on the value of thromboelastometry in the management of posttransplant complications is available.

Our study provides new insights into the complex haemostasis abnormalities involved in the pathogenesis of SOS/VOD by the use of thromboelastometry. The preliminary results of our ROTEM analyses indicated that a delay in the activation of coagulation initiated by intrinsic and extrinsic activators, fibrin formation and fibrin polymerisation, independent of platelets in INTEM, EXTEM and FIBTEM assay on day +12 , could be used to predict the occurrence of SOS/VOD in patients with acute leukaemia undergoing allogeneic HSCT. When compared to patients without SOS/VOD, patients with SOS/VOD had higher CT values in INTEM, EXTEM and FIBTEM assay, all suggesting impairment of fibrin polymerisation leading to abnormal clot structure and subsequently some defects in fibrinolysis. Complex haemostasis abnormalities including decreased factor VII activity, low thrombocytes count, abnormal expression of adhesion molecules, 
cytokines and procoagulant factors, together with the inhibition of anticoagulation pathway with decreased activity of antithrombin and protein $\mathrm{C}$, and the concurrent inhibition of fibrinolysis by the increased level of PAI-1, all together may contribute to the impairment of thrombin formation and may influence the results of the whole blood thromboelastometry. In our study group, other ROTEM parameters concerning the speed at which a solid clot forms (CFT), clot firmness (MCF) and clot lysis (ML), together with velocity profile, were unable to differentiate between patients with, or without, SOS/VOD symptoms on day +12 . This may be due to complex haemostasis impairment and the limited number of participants. However, on day +28 abnormalities in INTEM assay indicating reduced clot firmness in SOS/VOD (+) group were found. Moreover, both lower median values of MCF (reflecting clot firmness) and longer median CFT values (impairment of fibrin polymerisation) in INTEM and EXTEM assays indicates a trend to increased bleeding in patients with SOS/VOD on day +28 .

Mild SOS/VOD is self-limiting and does not require specific treatment. Therapies for SOS/VOD of moderate severity are mostly supportive, with diuretics and fluid restriction, as was done in our cohort. The only agent approved for the treatment of severe SOS/VOD is
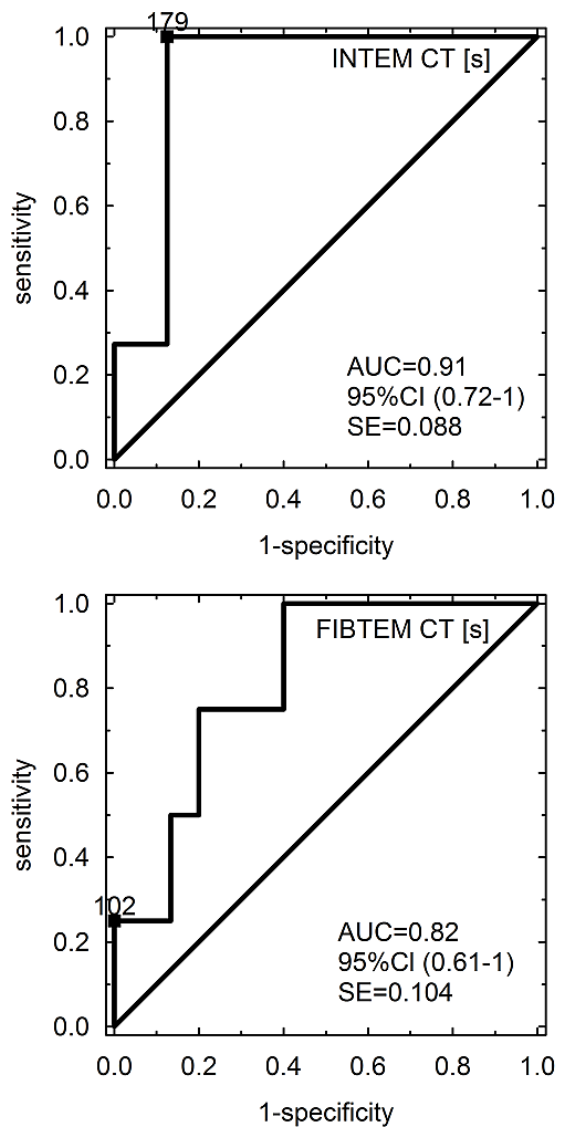

defibrotide [6]. Defibrotide which, among other functions, stabilises endothelial cells by reducing endothelial-cell activation and damage, also reduces plasma levels of PAI1 and results in the restoration of the thrombo-fibrynolitic balance $[36,38]$. It has been shown that not only delay in the initiation of defibrotide has been associated with worse outcome [41, 42], but also early intervention is justifiable and effective [14, 38]. Therefore, early diagnosis and treatment of selected patients of mild to moderate SOS/ VOD is essential to prevent disease progression and the development of severe SOS/VOD, and may possibly reduce mortality [6]. Defibrotide was not used on our patients, however, it is a treatment of first choice. Our findings regarding the thromboelastometry profile may allow for the determining of the optimal timing of the treatment of these patients but further studies are required.

The management of complex haemostasis abnormalities after HSCT is a challenge, not only because the patients are at increased risk of bleeding and thrombotic complications, but also because conventional coagulation tests may be unsuitable in the assessment of haemostasis. APTT and PT, did not differ significantly between our patients with, or without, SOS/VOD in the present study. This observation is in line with other studies evaluating the effects of defibotide on SOS/VOD [15, 45],

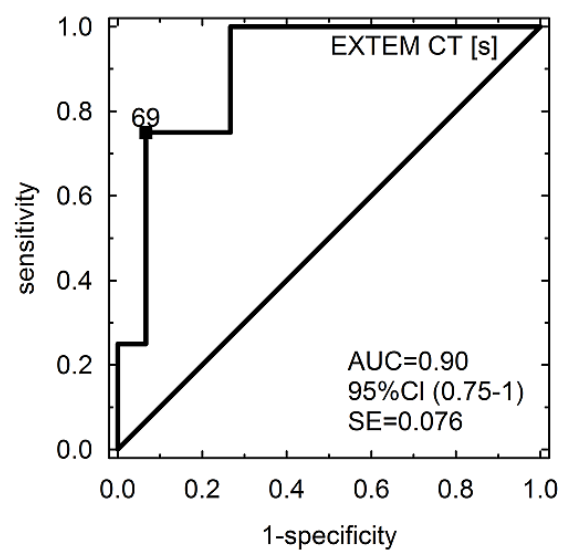

Figure 2: ROC curves of INTEM-CT, EXTEM-CT and FIBTEM-CT for SOS/VOD prediction. Abbreviations: CT clotting time [s]; AUC - area under the curve; CI - confidence interval; SE - standard error. 
Table 6: Grade of SOS severity [5-7]

\begin{tabular}{|c|c|c|c|}
\hline \multirow{2}{*}{ Clinical data } & \multicolumn{3}{|c|}{ SOS Grade } \\
\hline & Mild* & Moderate $^{*}$ & Severe $^{*}$ \\
\hline Bilirubin (mmol/l) & $34.2-51.3$ & $53-85.5$ & $>85.5$ \\
\hline Liver function tests ${ }^{* *}$ & $<3 \times$ normal & $3-5 \times$ normal & $>5 \times$ normal \\
\hline Weight above baseline & $2 \%$ & $2.1-5 \%$ & $>5 \%$ \\
\hline Renal function ${ }^{* * *}$ & Normal & $<2 \times$ normal & $\geq 2 \times$ normal \\
\hline Rate of change, days & $\begin{array}{c}\text { Slow } \\
\text { (over 6-7 days) }\end{array}$ & $\begin{array}{c}\text { Moderate } \\
\text { (over } 4-5 \text { days) }\end{array}$ & $\begin{array}{c}\text { Rapid } \\
\text { (over } 2-3 \text { days) }\end{array}$ \\
\hline
\end{tabular}

*Where two or more of the listed parameters are found.

** Serum aminotransferases.

${ }^{* * *}$ Serum creatinine.

in which most of the patients showed normal ranges of PT, APTT and fibrinogen but contrary to the results of Sartori et al. [43]. In the light of these data, it is possible that not only may ROTEM assessment be useful in the prediction of SOS/VOD occurrence but may also be appropriate to monitor the treatment with defibrotide or other agents (for example shortening of CT in ROTEM assays) or may assist in determining the optimal management of patients with SOS/VOD.

Although all our patients were at high risk of developing SOS/VOD, the incidence reached $26 \%$ in our study cohort (7/27), including mild disease - an incidence which is still within the range described in literature. The high rate of SOS/VOD documented in the early phases of HSCT was related to the high number of patients who had one or more conditions associated with a significant increased risk of developing SOS/VOD. Identification of the risk factors associated for the development of SOS/VOD is essential for adequate monitoring and early intervention and therapy [6]. Our data show that patients with pre-transplant ferritin levels above 1000 $\mathrm{ng} / \mathrm{dl}$ had an increased risk of SOS/VOD development which is consistent with literature [45-47]. Due to the limited number of patients studied, we were not able to demonstrate that other recently described patient-related or transplant-related factors had an impact on SOS/VOD occurrence. Most of the identified risk factors were found in either paediatric or adult patient populations, which were sometimes heterogeneous with regard to underlying disease and chemotherapeutic conditioning regimens.

Our study has some limitations. The number of participants was small, none of our patients had episodes of life-threatening bleeding or thrombotic events during the study and none required surgical procedures.

In conclusion, the baseline characteristics of haemostasis defects, as determined by thromboelastometry in whole blood, may be important in understanding the pathophysiology involved in SOS/VOD development by identification of specific biomarkers in ROTEM assay, indicative of SOS/ VOD occurrence. Our preliminary results provide new insights into the haemostasis abnormalities in adult patients undergoing HSCT for acute leukemia, with regard to SOS/VOD symptoms. In the SOS/VOD $(+)$ patients we have demonstrated a significant delay in the initiation of thrombin formation in the analysed ROTEM assays. The question of whether our findings may assist in the better management of patients undergoing HSCT should be clarified in a further prospective study.

\section{MATERIALS AND METHODS}

\section{Patients}

Twenty-seven adult with acute leukaemia, qualified for allo-HSCT between June 2011 and June 2012, were included in the study.

The diagnosis of SOS/VOD was established using the Baltimore criteria (total bilirubin $\geq 2.0 \mathrm{mg} / \mathrm{dL}$ with $\geq 2$ of the following: hepatomegaly, ascites or 5\% weight gain. Doppler ultrasound was performed to confirm clinical findings (hepatomegaly and ascites) and for differential diagnoses [14]. The clinical evaluation of SOS/VOD severity was performed retrospectively based on measurable clinical data including assessment of the rate of changes in the level of bilirubin, liver and renal function tests, and the amount and pace of weight gain above baseline, as proposed by Chao, with Carreras modification (Table 6) [5-7]. Clinical and laboratory assessment during hospital stay was performed daily.

Resolution of SOS/VOD was defined as normalization of serum bilirubin, resolution of ascites, and return of body weight to baseline values [15]. Based on the published data related to transplant- and/or patient- and disease-related factors for the development of SOS/VOD after HSCT, the following data were analysed: age, recipient gender, type of leukaemia and its stage, myeloablative conditioning (busulfan- or total body irradiation (TBI)-based), alternative donor, iron overload (pre-transplant ferritin level above $1000 \mathrm{ng} / \mathrm{dl}$ ), previous HSCT and liver disease [1, 5, 6]. 
Neutrophil engraftment was defined as an increase in an absolute neutrophil count greater than, or equal to, 0.5 $\mathrm{G} / 1$ for the first three consecutive days post-HSCT. Acute GvHD was diagnosed according to the IBMTR criteria $[16,17]$ and diagnosis of chronic GvHD was based on the NIH consensus criteria [18]. Organ toxicity was assessed according to the Common Terminology Criteria for Adverse Events (CTCAE). A serious bleeding event was defined as one that was life-threatening or resulted in prolonged hospitalization and occurring within 30 days after HSCT [19]. Bloodstream infection was defined as the isolation of a bacterial pathogen from at least one blood culture [20]. Advanced disease was defined as acute leukaemia beyond second complete remission (CR) or disease relapse.

\section{Thromboelastometry}

Fasting blood samples were collected for coagulation testing into vacuum tubes with minimal stasis (2.9 ml S-Monovette ${ }^{\circledR}$ Coagulation 9NC/3 ml, 3.2\% Sodium Citrate, Sarstedt, Nümbrecht, Germany). Rotation thromboelastometry was performed by a ROTEM ${ }^{\circledR}$ coagulation analyser according to the manufacturer's instructions (ROTEM ${ }^{\circledR}$ Gamma, Pentapharm, Munich, Germany) as previously described [21]. Four standard ROTEM assays, including INTEM, EXTEM, FIBTEM and APTEM were conducted before the conditioning regimen start (day -10$)$, on the day of stem cell infusion (day 0 ) and on day +12 and +28 after HSCT. The INTEM and EXTEM assays represent the contact system (or intrinsic coagulation pathway), and tissue factor activation (or extrinsic coagulation pathway), respectively. FIBTEM allows for the qualitative analysis of the fibrinogen levels and fibrin polymerisation independent of platelets, and is performed as EXTEM with inhibition of platelets by cytochalasin D. The APTEM assay is carried out to evaluate fibrinolysis using a fibrinolysis inhibitor (aprotinin). The following parameters were assessed: clotting time (CT), clot formation time (CFT), $\alpha$-angle, maximum clot firmness (MCF), amplitude at 10, 15, 20 and 25 minutes (A10, A15, A20, A25), maximum lysis (ML) and lysis index at $30 \mathrm{~min}$ (LI 30), $45 \mathrm{~min}$ (LI 45) and $60 \mathrm{~min}$ (LI 60). Assessment of the coagulation dynamic properties was performed as the first derivative of the thromboelastometry curves (velocity profile), as described by Sorensen et al. [22]. For all tests, the recorded ROTEM parameters of velocity profile were: MaxVel, t-MaxVel and the area under the velocity curve (AUC). All coagulation tests and ROTEM tests were performed within 30 minutes of sample collection.

To find the influence of platelets, fibrinogen and routine coagulation tests (activated partial thromboplastin time and protrombin time) on the ROTEM parameters, a full blood count with platelet count $\left(\times 10^{9} / 1\right)$, routine coagulation tests and plasma fibrinogen were determined by routine laboratory techniques (Instrumentation Laboratory Company - Bedford USA).

The Bioethical Committee of Poznan University of Medical Sciences approved the study and the patients provided their written informed consent, in accordance with the Declaration of Helsinki.

\section{Statistical methods}

The results are presented using methods of descriptive statistics such as frequency (n), medians and standard error (SE) or constant with interquartile ranges (IQRs: 25\%-75\%) and non-outlier. The Shapiro-Wilk test was performed to assess normality. In order to compare differences between the groups, the chi-square test or Fisher's exact test was used for categorical variables and the Mann-Whitney U test for continuous variables.

The ability of each single ROTEM parameter to discriminate between patients with, or without, SOS/VOD was also evaluated with receiver operating characteristic (ROC) curve analysis. For each parameter, the area under the ROC curve (AUC) was identified to determine the sensitivity and specificity with a $95 \%$ confidence interval (CIs) at cutoff values for the analysed parameter predictive of SOS/VOD occurrence. A $p$-value below 0.05 was regarded as statistically significant. The statistical analyses were performed with STATISTICA 10 and STATISTICA Medical Package 2.0 (StatSoft, Inc. 2012 software, Tulusa, USA).

ACKNOWLEDGMENTS AND FUNDING

We are grateful to Professor Geoffrey Shaw for his language editing of this paper.

\section{CONFLICTS OF INTEREST}

The authors declare no conflicts of interest.

\section{REFERENCES}

1. Mohty M, Malard F, Abecassis M, Aerts E, Alaskar AS, Aljurf M, Arat M, Bader P, Baron F, Bazarbachi A, Blaise D, Ciceri F, Corbacioglu S, et al. Sinusoidal obstruction syndrome/veno-occlusive disease: current situation and perspectives-a position statement from the European Society for Blood and Marrow Transplantation (EBMT). Bone Marrow Transplant. 2015; 50:781-89.

2. Coppell JA, Richardson PG, Soiffer R, Martin PL, Kernan NA, Chen A, Guinan E, Vogelsang G, Krishnan A, Giralt S, Revta C, Carreau NA, Iacobelli M, et al. Hepatic veno-occlusive disease following stem cell transplantation: incidence, clinical course, and outcome. Biol Blood Marrow Transplant. 2010; 16:157-68.

3. McDonald GB, Sharma P, Matthews DE, Shulman HM, Thomas ED. Venocclusive disease of the liver after 
bone marrow transplantation: diagnosis, incidence, and predisposing factors. Hepatology. 1984; 4:116-22.

4. Jones RJ, Lee KS, Beschorner WE, Vogel VG, Grochow LB, Braine HG, Vogelsang GB, Sensenbrenner LL, Santos GW, Saral R. Venoocclusive disease of the liver following bone marrow transplantation. Transplantation. 1987; 44:778-83.

5. Carreras E. How I manage sinusoidal obstruction syndrome after haematopoietic cell transplantation. Br J Haematol. 2015; 168:481-91.

6. Dalle JH, Giralt SA. Hepatic veno-occlusive disease after hematopoietic stem cell transplantation: risk factors and stratification, prophylaxis, and treatment. Biol Blood Marrow Transplant. 2016; 22:400-9.

7. Chao N. How I treat sinusoidal obstruction syndrome. Blood. 2014; 123:4023-26.

8. Akil A, Zhang Q, Mumaw CL, Raiker N, Yu J, Velez de Mendizabal N, Haneline LS, Robertson KA, Skiles J, Diaz-Ricart M, Carreras E, Renbarger J, Hanash S, et al. Biomarkers for diagnosis and prognosis of sinusoidal obstruction syndrome after hematopoietic cell transplantation. Biol Blood Marrow Transplant. 2015; 11:00460-7.

9. Carreras E, Diaz-Beya M, Rosinol L, Martinez C, Fernandez-Aviles F, Rovira M. The incidence of venoocclusive disease following allogeneic hematopoietic stem cell transplantation has diminished and the outcome improved over the last decade. Biol Blood Marrow Transplant. 2011; 17:1713-20.

10. Park YD, Yasui M, Yoshimoto T, Chayama K, Shimono T, Okamura T, Inoue M, Yumura-Yagi K, Kawa-Ha K. Changes in hemostatic parameters in hepatic veno-occlusive disease following bone marrow transplantation. Bone Marrow Transplant. 1997; 19:915-20.

11. Tanikawa S, Mori S, Ohhashi K, Akiyama H, Sasaki T, Kaku H, Hiruma K, Matsunaga T, Morita T, Sakamaki H. Predictive markers for hepatic veno-occlusive disease after hematopoietic stem cell transplantation in adults: a prospective single center study. Bone Marrow Transplant. 2000; 26:881-86.

12. Lee JH, Lee KH, Kim S, Lee JS, Kim WK, Park CJ, Chi HS, Kim SH. Relevance of proteins $\mathrm{C}$ and $\mathrm{S}$, antithrombin III, von Willebrand factor, and factor VIII for the development of hepatic veno-occlusive disease in patients undergoing allogeneic bone marrow transplantation: a prospective study. Bone Marrow Transplant. 1998; 22:883-8.

13. Lier $\mathrm{H}$, Vorweg $\mathrm{M}$, Hanke $\mathrm{A}$, Gorlinger $\mathrm{K}$. Thromboelastometry guided therapy of severe bleeding. Essener Runde algorithm. Hamostaseologie. 2013; 33:51-61.

14. Mohty M, Malard F, Abecassis M, Aerts E, Alaskar AS, Aljurf M, Arat M, Bader P, Baron F, Bazarbachi A, Blaise D, Ciceri F, Corbacioglu S, et al. Revised diagnosis and severity criteria for sinusoidal obstruction syndrome/ veno-occlusive disease in adult patients: a new classification from the European Society for Blood and Marrow Transplantation. Bone Marrow Transplant. 2016; 16:130.

15. Park M, Park HJ, Eom HS, Kwon YJ, Park JA, Lim YJ, Yoon JH, Kong SY, Ghim TT, Lee HW, Yun T, Park BK. Safety and effects of prophylactic defibrotide for sinusoidal obstruction syndrome in hematopoietic stem cell transplantation. Ann Transplant. 2013; 18:36-42.

16. Przepiorka D, Weisdorf D, Martin P, Klingemann HG, Beatty P, Hows J, Thomas ED. 1994 Consensus conference on acute GVHD grading. Bone Marrow Transplant. 1995; 15:825-28.

17. Rowlings PA, Przepiorka D, Klein JP, Gale RP, Passweg JR, Henslee-Downey PJ, Cahn JY, Calderwood S, Gratwohl A, Socie G, Abecasis MM, Sobocinski KA, Zhang MJ, et al. IBMTR Severity Index for grading acute graft-versus-host disease: retrospective comparison with Glucksberg grade. Br J Haematol. 1997; 97:855-64.

18. Filipovich AH, Weisdorf D, Pavletic S, Socie G, Wingard JR, Lee SJ, Martin P, Chien J, Przepiorka D, Couriel D, Cowen EW, Dinndorf P, Farrell A, et al. National Institutes of Health consensus development project on criteria for clinical trials in chronic graft-versus-host disease: I. Diagnosis and staging working group report. Biol Blood Marrow Transplant. 2005; 11:945-56.

19. Holler E, Kolb HJ, Greinix H, Perrotin D, Campilho F, Aversa F, Gil L, Cornelissen J, Varanese L, Schacht A, Friese A, Rustige J. Bleeding events and mortality in SCT patients: a retrospective study of hematopoietic SCT patients with organ dysfunctions due to severe sepsis or GVHD. Bone Marrow Transplant. 2009; 43:491-97.

20. Gil L, Poplawski D, Mol A, Nowicki A, Schneider A, Komarnicki M. Neutropenic enterocolitis after high-dose chemotherapy and autologous stem cell transplantation: incidence, risk factors, and outcome. Transpl Infect Dis. 2013; 15:1-7.

21. Rupa-Matysek J, Trojnarska O, Gil L, SzczepaniakChichel L, Wojtasinska E, Tykarski A, Grajek S, Komarnicki M. Assessment of coagulation profile by thromboelastometry in adult patients with cyanotic congenital heart disease. Int J Cardiol. 2016; 202:556-60.

22. Sorensen B, Ingerslev J. Whole blood clot formation phenotypes in hemophilia A and rare coagulation disorders. Patterns of response to recombinant factor VIIa. J Thromb Haemost. 2004; 2:102-10.

23. Nurnberger W, Michelmann I, Burdach S, Gobel U. Endothelial dysfunction after bone marrow transplantation: increase of soluble thrombomodulin and PAI-1 in patients with multiple transplant-related complications. Ann Hematol. 1998; 76:61-5.

24. Cutler C, Kim HT, Ayanian S, Bradwin G, Revta C, Aldridge J, Ho V, Alyea E, Koreth J, Armand P, Soiffer R, Ritz J, Richardson PG, et al. Prediction of veno-occlusive disease using biomarkers of endothelial injury. Biol Blood Marrow Transplant. 2010; 16:1180-85. 
25. Park YD, Yoshioka A, Kawa K, Ishizashi H, Yagi H, Yamamoto Y, Matsumoto M, Fujimura Y. Impaired activity of plasma von Willebrand factor-cleaving protease may predict the occurrence of hepatic veno-occlusive disease after stem cell transplantation. Bone Marrow Transplant. 2002; 29:789-94.

26. Ferra C, de Sanjose S, Gallardo D, Berlanga JJ, Rueda F, Marin D, de la Banda E, Ancin I, Peris J, Garcia J, Granena A. IL-6 and IL-8 levels in plasma during hematopoietic progenitor transplantation. Haematologica. 1998; 83:1082-87.

27. Iguchi A, Kobayashi R, Yoshida M, Kobayashi K, Matsuo K, Kitajima I, Maruyama I. Vascular endothelial growth factor (VEGF) is one of the cytokines causative and predictive of hepatic veno-occlusive disease (VOD) in stem cell transplantation. Bone Marrow Transplant. 2001; 27:1173-80.

28. Heikinheimo M, Halila R, Fasth A. Serum procollagen type III is an early and sensitive marker for veno-occlusive disease of the liver in children undergoing bone marrow transplantation. Blood. 1994; 83:3036-40.

29. Eltumi M, Trivedi P, Hobbs JR, Portmann B, Cheeseman P, Downie C, Risteli J, Risteli L, Mowat AP. Monitoring of veno-occlusive disease after bone marrow transplantation by serum aminopropeptide of type III procollagen. Lancet. 1993; 342:518-21.

30. Rio B, Bauduer F, Arrago JP, Zittoun R. N-terminal peptide of type III procollagen: a marker for the development of hepatic veno-occlusive disease after BMT and a basis for determining the timing of prophylactic heparin. Bone Marrow Transplant. 1993; 11:471-72.

31. Scrobohaci ML, Drouet L, Monem-Mansi A, Devergie A, Baudin B, D’Agay MF, Gluckman E. Liver veno-occlusive disease after bone marrow transplantation changes in coagulation parameters and endothelial markers. Thromb Res. 1991; 63:509-19.

32. Faioni EM, Krachmalnicoff A, Bearman SI, Federici AB, Decarli A, Gianni AM, McDonald GB, Mannucci PM. Naturally occurring anticoagulants and bone marrow transplantation: plasma protein $\mathrm{C}$ predicts the development of venocclusive disease of the liver. Blood. 1993; 81:3458-62.

33. Iguchi A, Kobayashi R, Kaneda M, Kobayashi K. Plasma protein $\mathrm{C}$ is a useful clinical marker for hepatic venoocclusive disease (VOD) in stem cell transplantation. Pediatr Blood Cancer. 2010; 54:437-43.

34. Tabbara IA, Ghazal CD, Ghazal HH. Early drop in protein $\mathrm{C}$ and antithrombin III is a predictor for the development of venoocclusive disease in patients undergoing hematopoietic stem cell transplantation. J Hematother. 1996; 5:79-84.

35. Salat C, Holler E, Kolb HJ, Reinhardt B, Pihusch R, Wilmanns W, Hiller E. Plasminogen activator inhibitor-1 confirms the diagnosis of hepatic veno-occlusive disease in patients with hyperbilirubinemia after bone marrow transplantation. Blood. 1997; 89:2184-88.
36. Kaleelrahman M, Eaton JD, Leeming D, Bowyer K, Taberner D, Chang J, Scarffe JH, Chopra R. Role of plasminogen activator inhibitor-1 (PAI-1) levels in the diagnosis of BMT-associated hepatic veno-occlusive disease and monitoring of subsequent therapy with defibrotide (DF). Hematology. 2003; 8:91-95.

37. Pihusch M, Wegner H, Goehring P, Salat C, Pihusch V, Hiller E, Andreesen R, Kolb HJ, Holler E, Pihusch R. Diagnosis of hepatic veno-occlusive disease by plasminogen activator inhibitor-1 plasma antigen levels: a prospective analysis in 350 allogeneic hematopoietic stem cell recipients. Transplantation. 2005; 80:1376-82.

38. Richardson PG, Riches ML, Kernan NA, Brochstein JA, Mineishi S, Termuhlen AM, Arai S, Grupp SA, Guinan EC, Martin PL, Steinbach G, Krishnan A, Nemecek ER, et al. Phase 3 trial of defibrotide for the treatment of severe venoocclusive disease and multi-organ failure. Blood. 2016; 29:2015-10.

39. Whiting D, DiNardo JA. TEG and ROTEM: technology and clinical applications. Am J Hematol. 2014; 89:228-32.

40. Hincker A, Feit J, Sladen RN, Wagener G. Rotational thromboelastometry predicts thromboembolic complications after major non-cardiac surgery. Crit Care. 2014; 18: 014-0549.

41. Corbacioglu S, Greil J, Peters C, Wulffraat N, Laws HJ, Dilloo D, Straham B, Gross-Wieltsch U, Sykora KW, Ridolfi-Luthy A, Basu O, Gruhn B, Gungor T, et al. Defibrotide in the treatment of children with venoocclusive disease (VOD): a retrospective multicentre study demonstrates therapeutic efficacy upon early intervention. Bone Marrow Transplant. 2004; 33:189-95.

42. Richardson PG, Triplett BM, Kernan NA, Grupp SA, Arai S, Haut PR, Martin PL, Symons HJ, Lehmann LE, SimmsWaldrip T, Gillio AP, Horn BN, Shore TB, et al. Results of the large prospective study on the use of defibrotide (DF) in the treatment of hepatic veno-occlusive disease (VOD) in hematopoietic stem cell transplant (HSCT). Early intervention improves outcome - updated results of a treatment IND (T-IND) expanded access protocol. Blood. 2013; 122:700.

43. Sartori MT, Cesaro S, Peruzzo M, Messina C, Saggiorato G, Calore E, Pillon M, Varotto S, Spiezia L, Cella G. Contribution of fibrinolytic tests to the differential diagnosis of veno-occlusive disease complicating pediatric hematopoietic stem cell transplantation. Pediatr Blood Cancer. 2012; 58:791-97.

44. Chalandon Y, Roosnek E, Mermillod B, Newton A, Ozsahin H, Wacker P, Helg C, Chapuis B. Prevention of veno-occlusive disease with defibrotide after allogeneic stem cell transplantation. Biol Blood Marrow Transplant. 2004; 10:347-54.

45. Morado M, Ojeda E, Garcia-Bustos J, Aguado MJ, Arrieta R, Quevedo E, Navas A, Hernandez-Navarro F. BMT: Serum ferritin as risk factor for veno-occlusive disease of the liver. Hematology. 2000; 4:505-12. 
46. Armand P, Kim HT, Cutler CS, Ho VT, Koreth J, Alyea EP, Soiffer RJ, Antin JH. Prognostic impact of elevated pretransplantation serum ferritin in patients undergoing myeloablative stem cell transplantation. Blood. 2007; 109:4586-88.

47. Maximova N, Ferrara G, Minute M, Pizzol A, Kiren V, Montico M, Gregori M, Tamaro P. Experience from a single paediatric transplant centre with identification of some protective and risk factors concerning the development of hepatic veno-occlusive disease in children after allogeneic hematopoietic stem cell transplant. Int J Hematol. 2014; 99:766-72. 